\title{
The VISA-A questionnaire: a valid and reliable index of the clinical severity of Achilles tendinopathy
}

J M Robinson, J L Cook, C Purdam, P J Visentini, J Ross, N Maffulli, J E Taunton, K M Khan, for the Victorian Institute of Sport Tendon Study Group
Department of Family Practice (Allan McGavin Sports Medicine Centre) and School of Human Kinetics, University of British Columbia, Vancouver, Canada J M Robinson

J E Taunton

K M Khan

Department of Physiotherapy, LaTrobe University, Bundoora, Australia and Victorian Institute of Sport Tendon Study Group, Melbourne, Australia

J L Cook

C Purdam

Alphington Sports

Medicine Clinic and

School of

Physiotherapy,

University of

Melbourne, Australia and Victorian Institute of Sport Tendon Study Group, Melbourne, Australia

P J Visentini

Department of Orthopaedic Surgery, University of

Aberdeen, Aberdeen, Scotland, UK

J Ross

N Maffulli

Correspondence to: Dr Khan, James Mather Building, 5804 Fairview Avenue, Vancouver V6T 1Z3, Canada

kkhan@interchange.ubc.ca

Accepted 9 July 2001

\begin{abstract}
Background-There is no disease specific, reliable, and valid clinical measure of Achilles tendinopathy.

Objective-To develop and test a questionnaire based instrument that would serve as an index of severity of Achilles tendinopathy.

Methods-Item generation, item reduction, item scaling, and pretesting were used to develop a questionnaire to assess the severity of Achilles tendinopathy. The final version consisted of eight questions that measured the domains of pain, function in daily living, and sporting activity. Results range from 0 to 100 , where 100 represents the perfect score. Its validity and reliability were then tested in a population of non-surgical patients with Achilles tendinopathy $(n=45)$, presurgical patients with Achilles tendinopathy (n $=14$ ), and two normal control populations (total $\mathbf{n}=87$ ).

Results-The VISA-A questionnaire had good test-retest $(r=0.93)$, intrarater (three tests, $r=0.90)$, and interrater $(r=0.90)$ reliability as well as good stability when compared one week apart $(r=0.81)$. The mean $(95 \%$ confidence interval) VISA-A score in the non-surgical patients was 64 (59-69), in presurgical patients 44 (28-60), and in control subjects it exceeded 96 (94-99). Thus the VISA-A score was higher in non-surgical than presurgical patients $(p=0.02)$ and higher in control subjects than in both patient populations $(p<0.001)$. Conclusions-The VISA-A questionnaire is reliable and displayed construct validity when means were compared in patients with a range of severity of Achilles tendinopathy and control subjects. The continuous numerical result of the VISA-A questionnaire has the potential to provide utility in both the clinical setting and research. The test is not designed to be diagnostic. Further studies are needed to determine whether the VISA-A score predicts prognosis.

(Br F Sports Med 2001;35:335-341)
\end{abstract}

Keywords: Achilles; tendinopathy; tendinitis; outcome; questionnaire

Achilles tendinopathy is a major cause of prolonged pain and disability in sportspeople and those who undertake an active lifestyle such as hiking and walking. ${ }^{1-3}$ It may cause
$2-16 \%$ of people to abandon the causative activity. ${ }^{1245}$ In some settings, $20-30 \%$ of patients who present with this condition require surgical treatment..$^{6-8}$ Furthermore, as physical inactivity is a risk factor for many multisystem diseases, ${ }^{9}$ Achilles tendinopathy can lead to suboptimal overall health, not just sporting inconvenience.

There have been many studies published on the subject of Achilles tendinopathy, but there remain very few prospective studies of treatment outcome. ${ }^{4}{ }^{10-13}$ One factor that limits efficacy studies in Achilles tendon research is the lack of a standardised outcome measure by which to evaluate treatment of Achilles tendinopathy. ${ }^{14}$

Several quantitative tests of ankle function ${ }^{15}$ have been used to measure outcome in Achilles tendinopathy, ${ }^{4}$ and there are tests to evaluate Achilles tendon function after complete rupture. ${ }^{16}$ However, condition specific numerical scales generally have greater sensitivity and specificity than general purpose scales. ${ }^{17-20}$ The inventor of the much used Lysholm scale for knee instability emphasised the need for "different or modified scoring systems for the follow-up of patients with different diagnoses". ${ }^{18}$ A specific scale for patients with patellar tendinopathy ${ }^{19}$ has proved useful in numerous peer reviewed studies. ${ }^{21-23}$ Therefore the aim of this study was to develop and test a questionnaire based instrument to measure the severity of Achilles tendinopathy.

\section{Methods}

To develop the questionnaire we performed item generation, item reduction, item scaling, and pretesting as outlined below. We then tested its validity and reliability in clinical and control populations. Ethics approval was obtained from the University of British Columbia ethics committee.

ITEM GENERATION

The Victorian Institute of Sport tendon study group (Melbourne, Australia) first developed an index of severity for patellar tendinopathy. ${ }^{19}$ We used this as a template from which to develop a questionnaire specifically for use in Achilles tendinopathy. The literature was reviewed to find items that would be appropriate for inclusion. In addition, colleagues were consulted to find unpublished items used in clinical practice. The second step involved the interview of colleagues with expertise in the area of Achilles tendinopathy. Finally, patients were informally interviewed about symptoms that they felt were important. 
IN THIS QUESTIONNAIRE, THE TERM PAIN REFERS SPECIFICALLY TO PAIN IN THE ACHILLES TENDON REGION

1. For how many minutes do you have stiffness in the Achilles region on first getting up?

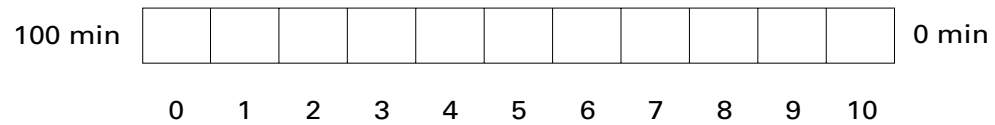

POINTS

2. Once you are warmed up for the day, do you have pain when stretching the Achilles tendon fully over the edge of a step? (keeping knee straight)

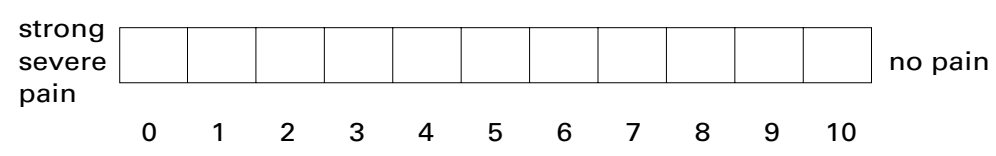

POINTS

3. After walking on flat ground for 30 minutes, do you have pain within the next 2 hours?

(If unable to walk on flat ground for 30 minutes because of pain, score 0 for this question).

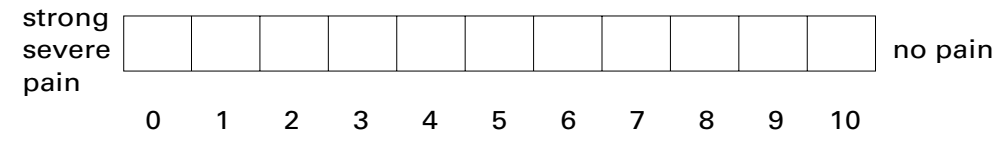

POINTS

Figure 1 The VISA-A questionnaire. Eight questions are used to determine the VISA-A score (continued). The VISA-A questionnaire can be dowloaded in full at http://bjsm.bmjjournals/cgi/content/full/35/5/336/DC1.

ITEM REDUCTION

A focus group consisting of the principal questionnaire developer, a primary care sports medicine doctor, and two physiotherapists reviewed the items generated. They decided that three domains-pain, functional status, and activity-were necessary, and they allocated three questions to each domain (fig 1). ${ }^{24-27}$

ITEM SCALING

A visual analogue scale (VAS) is more accurate and sensitive than categorical verbal scales. ${ }^{25} 28-$ ${ }_{32}$ The first six questions use a VAS so that the patient may report magnitude of a continuum of subjective symptoms. Activity is best measured using a categorical rating system based on an incremental range of values. ${ }^{33}$ Thus the final two questions used a categorical rating scale.

PRETESTING

Before being shown the working version of the questionnaire, a group of 15 clinicians expert in the field of tendon injuries were asked to identify questions they felt were important in assessing the severity of Achilles tendon disorders. The group comprised eight physiotherapists, four primary care doctors, one orthopaedic surgeon, and one rehabilitation specialist.

The same 15 participants were then shown the VISA-A score and asked to evaluate the questionnaire. They were specifically asked if there were any questions they would add, delete, or modify. Fourteen of the participants had no questions to add, and none wanted any deleted or modified.

\section{WEIGHTING}

The questionnaire tests the three significant domains of dysfunction with three questions for each (question 8 is effectively two questions, one relating to pain with activity and the other to duration of activity). By removing redundancies and eliminating items of less importance, weighting of the remaining items may be the same (each question is scored out of 10) without affecting the value of the questionnaire. ${ }^{20}$ The final version of the questionnaire 


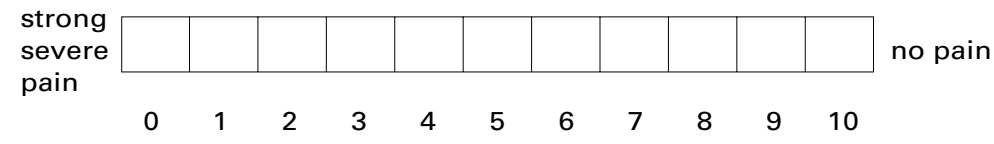

POINTS

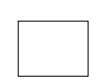

5. Do you have pain during or immediately after doing 10 (single leg) heel raises from a flat surface?

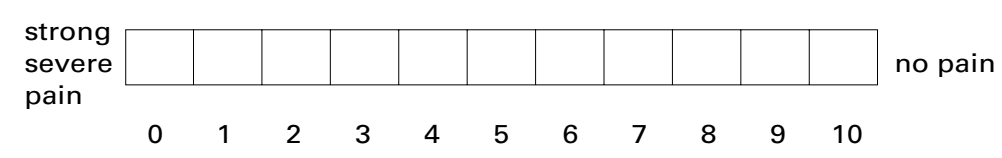

POINTS

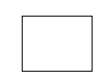

6. How many single leg hops can you do without pain?

0

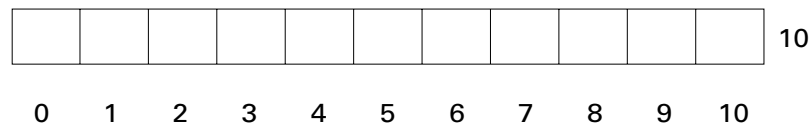

POINTS

10

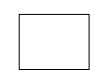

7. Are you currently undertaking sport or other physical activity?

0

Not at all

POINTS

4

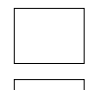

Modified training \pm modified competition

7

Full training \pm competition but not at same level as when symptoms began

10

Competing at the same or higher level as when symptoms began

Figure 1 continued

was called the Victorian Institute of Sport Assessment-Achilles questionnaire (VISA-A) (fig 1).

RELIABILITY AND VALIDITY TESTING

We administered the VISA-A questionnaire to four populations: group 1 (non-surgical patients; $n=45$ ) attended a primary care sports medicine clinic; group 2 (presurgical patients; $\mathrm{n}=14$ ) had been referred to a sports orthopaedist for tendon surgery; group 3 (university students; $n=63$ ) represented a convenience sample of young normally active people to serve as a control group; group 4 (members of a running club; $\mathrm{n}=24$ ) represented active, but non-injured people whose age matched the patient groups. As imaging does not provide a yardstick for tendon disorders, diagnosis was by assessment of two expert clinicians, as has been justified in other tendon studies. ${ }^{34-36}$ For inclusion in the study, subjects in all groups had to be older than 18 who were able to give written informed consent. For groups 1 and 2, subjects had to have a diagnosis of Achilles tendinosis, paratendinitis, or partial rupture with or without a retrocalcaneal or Achilles bursitis. For groups 3 and 4, subjects had to belong to the two groups as defined. Women who were pregnant or nursing were excluded, as were patients with a total rupture of the Achilles tendon. Subjects with previous or current Achilles tendon symptoms, but who were not currently undergoing treatment for the condition, were not excluded from the control groups as these groups were designed to reflect the populations.

We tested construct validity in two ways. Firstly, the 45 non-surgical patients in group 1 completed the VISA-A test and two other 
8. Please complete EITHER A, B or $\mathbf{C}$ in this question.

- If you have no pain while undertaking Achilles tendon loading sports please complete Q8A only.

- If you have pain while undertaking Achilles tendon loading sports but it does not stop you from completing the activity, please complete Q8B only.

- If you have pain that stops you from completing Achilles tendon loading sports, please complete $\mathbf{Q 8 C}$ only.

A. If you have no pain while undertaking Achilles tendon loading sports, for how long can you train/practise?

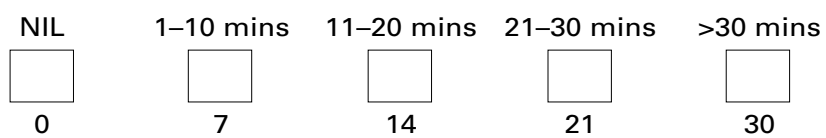

If you have some pain while undertaking Achilles tendon loading sports, but it does not stop you from completing your training/practice, for how long can you train/practise?
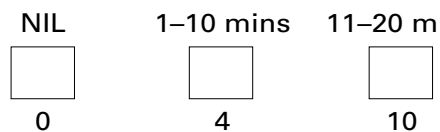

10
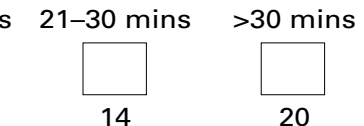

OR

C. If you have pain that stops you from completing your training/practice in Achilles tendon loading sports, for how long can you train/practise?
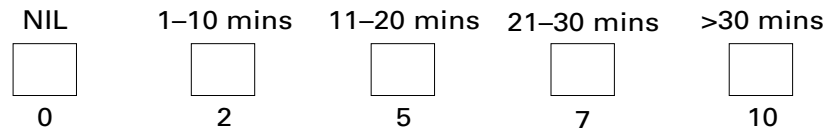

generic tendon grading systems ${ }^{37} 38$ at one visit. Secondly, we tested the VISA-A scale in both surgical patients (group 2) who are generally considered to have the most significant degree of disease, and two control populations (groups 3 and 4).

All patients in group 1 attended our research centre on two occasions one week apart and completed the VISA-A questionnaire three times to test its reliability. At either the first or second visit, patients completed the questionnaire twice with a 60 minute interval to measure test-retest reliability. Short term reliability was measured by comparing VISA-A scores at one week follow up with the baseline measure. To test intertester reliability, the chief investigator (JR) and one of two other trained researchers administered the VISA-A questionnaire in a subset of 16 subjects. We also tested intertester and test-retest reliability studies in a control group (group 4). We did not test reliability in the surgical patients as they travelled to a tertiary referral centre and were not available for the one week follow up (short term stability) measure.

\section{STATISTICAL METHODS}

All data were entered on a personal computer and results analysed using SPSS Version 7.0. Descriptive data are reported as mean, standard deviation, and 95\% confidence interval. Correlation of VISA-A scores in group 1 patients with the other scoring scales was by Spearman's rank correlation coefficient for non-parametric data, as the data were not normally distributed. Reliability data were analysed by Pearson's $r$, as these data were normally distributed. VISA-A scores in the various study groups were compared using single factor analysis of variance with Tukey's post hoc test. 
Table 1 Descriptive characteristics of the study population

\begin{tabular}{|c|c|c|c|c|c|c|c|c|c|}
\hline & \multicolumn{3}{|c|}{ Age (years) } & \multicolumn{3}{|c|}{ Duration of symptoms (months) } & \multicolumn{3}{|c|}{ VISA-A score } \\
\hline & Mean & $S D$ & $95 \% C I$ & Mean & $S D$ & $95 \% C I$ & Mean & $S D$ & $95 \% C I$ \\
\hline Group $1(n=45,18 F, 27 M)$ & 42.3 & 11.4 & 38.9 to 45.7 & 21.0 & 25.5 & 7.7 to 23.1 & 64 & 17 & 59 to 69 \\
\hline Group $2(\mathrm{n}=14,6 \mathrm{~F}, 8 \mathrm{M})$ & 44.3 & 14.8 & 35.4 to 53.3 & 19.2 & 4.1 & 14.8 to 19.2 & 44 & 28 & 28 to 60 \\
\hline Group $3(n=63,31 F, 32 M)$ & 23.0 & 2.9 & 22.3 to 23.7 & \multicolumn{3}{|c|}{$\mathrm{N} / \mathrm{A}^{\star}$} & 96 & 7 & 94 to 98 \\
\hline Group $4(\mathrm{n}=20,9 \mathrm{~F}, 11 \mathrm{M})$ & 40.9 & 9.1 & 38.7 to 43.1 & \multicolumn{3}{|c|}{$\mathrm{N} / \mathrm{A}^{\star}$} & 98 & 3 & 97 to 99 \\
\hline
\end{tabular}

*Although a total of five control subjects had Achilles tendon symptoms for 0.5-2 months, they are not tabled here as they are not representative of the group.

\section{Results}

SUBJECTS

Table 1 shows descriptive data on the study participants. Twelve group 1 non-surgical patients (24 tendons) had bilateral symptoms, and 33 patients had unilateral symptoms, giving a total of 57 symptomatic tendons and 33 asymptomatic tendons. As the presence of Achilles tendon pain was not an exclusion from any group in the study, two university students and three subjects from running clubs reported pain in the Achilles tendon.

QUESTIONNAIRE

The final version of the VISA-A questionnaire contained eight questions that covered the three domains of pain (questions 1-3), function (questions 4-6), and activity (questions 7 and 8.) Questions one to seven are scored out of 10 , and question 8 carries a maximum of 30 . Scores are summed to give a total out of 100 . An asymptomatic person would score 100 (fig 1). For question 8, participants must answer only part $\mathrm{A}, \mathrm{B}$, or $\mathrm{C}$. If the participant has pain when undertaking sport, he or she automatically loses at least 10 , and possibly 20 , points.

\section{RELIABILITY}

Table 2 summarises the reliability of the VISA-A questionnaire. There was no difference in scores whether the test-retest questionnaires were completed at the first visit or at the second visit $(\mathrm{p}=0.58)$.

\section{VALIDITY}

Figures 2 and 3 illustrate the construct validation. The VISA-A score correlated significantly with both Percy and Conochie's grade of severity (Spearman's $r=0.58 ; \mathrm{p}<0.01$ ) and that of Curwin and Stanish (Spearman's $r=$ $-0.57 ; \mathrm{p}<0.001)$.

The second part of construct validity testing compared scores in various patient and control groups. Comparing the VISA-A scores of all four groups showed that the mean VISA-A scores of the groups of patients with Achilles tendinopathy (both group 1 and 2) were significantly lower $(p<0.001)$ than those of the control groups (both group 3 and 4) (fig 4). Furthermore, patients with Achilles tendinopathy in

Table 2 Summary of reliability of VISA-A score

\begin{tabular}{llllll}
\hline & \multicolumn{2}{c}{ Group 1, non-surgical patients } & & \multirow{2}{*}{ Group 4, running club } \\
\cline { 2 - 3 } \cline { 5 - 6 } Reliability (2 tests except where stated) & $n$ & Pearson's $r$ & & $n$ & Pearson's $r$ \\
\hline Test-retest reliability & 45 & 0.93 & & 24 & 0.98 \\
Intrarater reliability (3 trials) & 29 & 0.90 & & $\overline{2}$ & - \\
Interrater reliability & 16 & 0.90 & & 24 & 0.97 \\
Short term (one week) reliability & 45 & 0.81 & & 12 & 0.98 \\
\hline
\end{tabular}

group 1 (non-surgical patients) had a significantly higher mean VISA-A score than patients in group 2 (presurgical patients) $p=0.02$ (fig 4).

\section{Discussion}

To be in a position to practice evidence based medicine, the sports medicine community

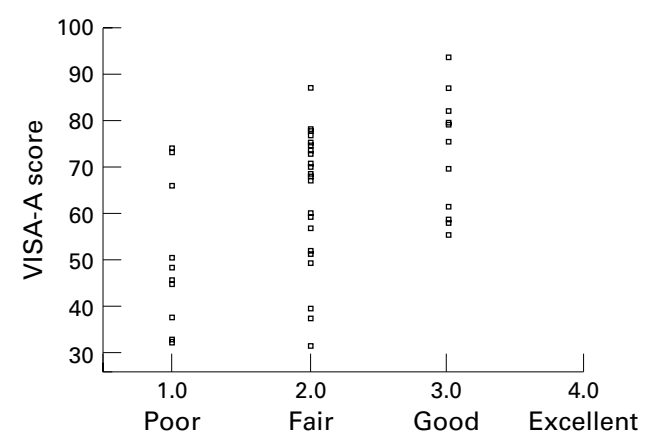

Figure 2 Scatter plot of VISA-A score compared with the grade of severity of Percy and Conochie. ${ }^{37}$

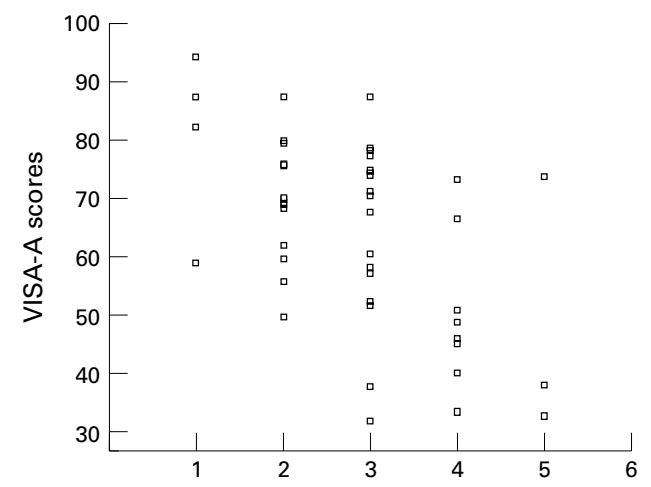

Figure 3 Scatter plot of VISA-A score compared with the grade of severity of Curwin and Stanish. ${ }^{38}$

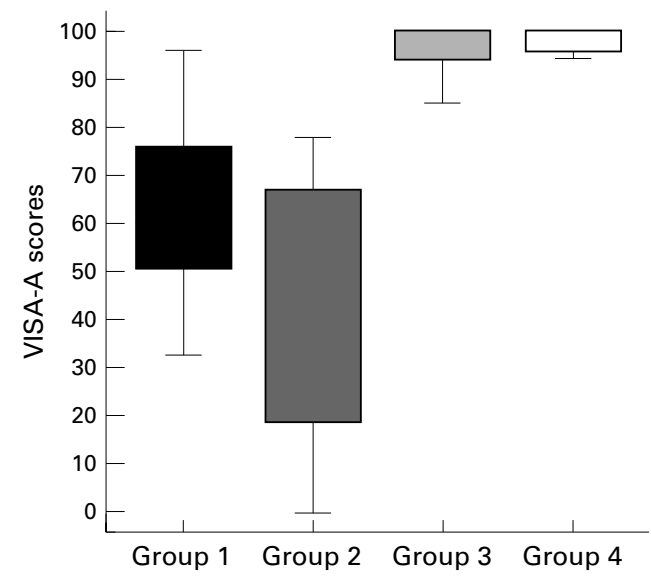

Figure 4 Box plot showing VISA-A scores among the four groups of participants in this study. 
must conduct studies with objective outcome measures. To date there is no tool designed for this purpose in patients with Achilles tendinopathy. This study suggests that the VISA-A questionnaire may fill this void, as it is valid, reliable, and user friendly.

STRENGTHS OF THE VISA-A QUESTIONNAIRE

The VISA-A questionnaire displayed construct validity when used in two populations of patients with Achilles tendinopathy and control subjects. The questionnaire avoids the redundant components of non-specific scoring systems such as that developed for hind foot problems by the American Orthopaedic Society, ${ }^{17}{ }^{27}$ and those devised for Achilles tendon rupture. ${ }^{39} 40$ The VISA-A questionnaire also compares favourably with two generic tendon grading systems ${ }^{37} 38$ that use a categorical rating scale. There are no published validation or reliability data for either of these scales and categorical scales have been criticised for being insensitive to subtle changes in a clinical condition. ${ }^{41}{ }^{42}$

We believe that the questionnaire's excellent reliability reflects the uncomplicated nature of the questions and the use of a VAS that has proven reliable in questionnaires. ${ }^{43}$ Because the questionnaire can be self administered with a minimum of investigator assistance, it avoids some potential for observer bias that can diminish interobserver reliability.

As well as being valid and reliable, the VISA-A questionnaire is easy to use. It generally takes less than five minutes to administer, even in patients with chronic and severe symptoms. Also, we found that a medical student and a sports medicine doctor obtained virtually identical results, so specialised training is not required to administer the test. In clinical practice, we have found that patients who have had the questionnaire administered once by an investigator or clinician can easily complete the questionnaire alone on future occasions. Although this aspect of questionnaire use was not tested formally in this study, we believe the instrument has the potential to be self administered after the baseline test.

The continuous numerical result of the VISA-A questionnaire is ideal for comparing patients' progress in the clinical setting. As tendinopathy takes some time to resolve, weeks can elapse between doctor visits. The VISA-A score could be used to monitor a patient's progress. However, until the VISA-A scale is tested in longitudinal studies, we can only suggest it has potential for use in this way.

In the research setting, this index of severity of Achilles tendinopathy could prove very useful in descriptive studies as well as providing an outcome measure in intervention studies. However, we consider that any such studies would be greatly strengthened if they were carried out in homogeneous groups of athletesfor example, runners only, volleyball players only-as the VISA-A score has not yet been shown to respond equally to change in Achilles tendon function in different sports. Nevertheless, our data show that it is suitable for patients being managed both conservatively and surgically. Because the test requires no equipment and is not subject to observer bias, it may prove suitable for data collected in multicentre studies.

CLINICALLY RELEVANT METHODOLOGICAL ISSUES WHEN ADMINISTERING THE VISA-A

We note in question 8 that participants answer only part A, B, or C. However, we found that, after we explained this concept to participants, they had no trouble understanding it subsequently. The question is scored in this way to differentiate patients who have a certain functional level without pain, and those who perform to a similar level of function despite pain. Clearly the latter is not as close to perfect as the former, and the VISA-A score reflects that because of the three stems of question 8 .

As a corollary of this point, the recreational person who has Achilles tendinopathy-for example, an archer-could only score a maximum of 70 on the VISA-A score as outlined. In this clinical setting, once the patient reached 70 , both the patient and the doctor would realise that this indicated that the patient was cured. In randomised controlled trials in which the VISA-A is used as an outcome measure, researchers could standardise the score as a percentage, or, as is most usual, report change, either in absolute units or as a percentage of baseline. Thus, just as knee extension strength (a measurement) is sometimes used as a raw score (in kg), adjusted for height in the analysis (in $\mathrm{kg} / \mathrm{cm}$ ), or corrected for in regression, so the VISA score has the potential to be used in a variety of ways depending on the setting.

Although there was a statistically significant difference between population mean VISA-A scores in the non-surgical and surgical patient groups, this does not mean that the VISA-A score has any role to play in the decision as to whether or not surgery is indicated. The only indication for surgery in Achilles tendinopathy is failure of conservative management, ${ }^{44}$ and this remains a clinical decision to be made between doctor and patient.

LIMITATIONS OF THE VISA-A QUESTIONNAIRE We emphasise that the VISA-A questionnaire is an index of the severity of a clinically diagnosed condition-it is not a diagnostic tool. Thus other conditions that influence lower limb function (such as ankle sprain) will reduce a patient's VISA-A score. Furthermore, limitation of function (such as severe sciatica) limits the subject's ability to score well in question 8, even though the Achilles tendon may be uninjured. This has not proved to be a problem in patellar tendon research ${ }^{21-23}$ that used a similarly designed VISA questionnaire for that condition (VISA-P).

A limitation of this study is that we do not have longitudinal change data, and therefore cannot comment on the sensitivity of the VISA-A to detect change with intervention. This aspect of the instrument will be evaluated in a future study. Further studies should also test the reliability and short term stability of the VISA score in the setting of a surgical population. However, we have no reason to suspect 
that it would be inferior to the reliability data reported here.

We conclude that the VISA-A questionnaire provides a valid, reliable, and user friendly index of the severity of Achilles tendinopathy. Although the limited time for consultation in routine clinical practice means that the main role of this tool is likely to be as an outcome measure in treatment studies, the VISA-A scale can be easily administered in clinical practice. Further studies are needed to determine whether the VISA-A score predicts prognosis.

This project was funded in part by a grant from the British Columbia Sports Medicine Research Foundation.

1 Kvist M. Achilles tendon injuries in athletes. Sports Med 1994;18:173-201.

2 Józsa L, Kannus P. Human tendons. Champaign, IL: Human Kinetics, 1997.

3 Alfredson H, Lorentzon R. Chronic Achilles tendinosis: recommendations for treatment and prevention. Sports Med 2000;29:135-46.

4 Paavola M, Kannus P, Paakkala T, et al. Long-term prognosis of patients with achilles tendinopathy. An observationa 8-year follow-up study. Am $\mathcal{F}$ Sports Med 2000;28:634-42.

5 Almekinders LC. Tendinitis and other chronic tendinopathies. I Am Acad Orthop Surg 1998;6:157-164.

6 Waterston SW, Maffulli N, Ewen SWB. Subcutaneous rupture of the Achilles tendon: basic science and some aspect of clinical practice. Brf Sports Med 1997;31:289-98.

7 Kvist M. Achilles tendon overuse injuries. A clinical and pathophysiological study in athletes with special reference to Achilles paratenonitis. $\mathrm{PhD}$ thesis, University of Turku, 1991.

8 Leppilahti J, Karpakka J, Gorra A, et al. Surgical treatment of overuse injuries to the Achilles tendon. Clinical fournal of Sports Medicine 1994;4:100-7.

9 Booth FW, Gordon SE, Carlson CJ, et al. Waging war on modern chronic diseases: primary prevention through modern chronic diseases: primary prevention

10 Maffulli N, Testa V, Capasso G, et al. Results of percutaneous longitudinal tenotomy in Achilles tendinopathy in ous longitudinal tenotomy in Achilles tendinopathy in 25:835-40

11 Alfredson H, Pietila T, Lorentzon R. Chronic Achilles tendinitis and calf muscle strength. Am $\mathcal{F}$ Sports $\mathrm{Med}$ $1996 ; 24: 829-33$

12 Alfredson H, Pietila T, Jonsson P, et al. Heavy-load eccentric calf muscle training for the treatment of chronic Achille tendinosis. Am $\mathcal{F}$ Sports Med 1998;26:360-6.

13 Mafi N, Lorentzon R, Alfredson H. Superior short-term results with eccentric calf muscle training compared to concentric training in a randomized prospective multicenter study on patients with chronic Achilles tendinosis. Knee Surg Sports Traumatol Arthrosc 2001;9:42-7.

14 Khan KM, Maffulli N. Tendinopathy: an Achilles' heel for athletes and clinicians. [Lead editorial].Clinical fournal of Sport Medicine 1998;8:151-4.

15 Kaikkonen A, Kannus P, Jarvinen M. A performance test protocol and scoring scale for the evaluation of ankle injuprotocol and scoring scale for the eva
ries. Am 7 Sports Med 1994;22:462-9.

16 Leppilahti J, Forsman K, Puranen J, et al. Outcome and Leppilahti J, Forsman K, Puranen J, et al. Outcome and
prognostic factors of achilles rupture repair using a new prognostic factors of achilles rupture repair usin
scoring method. Clin Orthop 1998;346:152-61.

17 Kitaoka HB, Patzer GL. Analysis of clinical grading scales fitaoka HB, Patzer GL. Analysis of clinical grading

18 Lysholm J, Gillquist J. The evaluation of knee ligament surgery with special emphasis to the use of a knee scoring scale. Am f Sports Med 1982;10:150-4.

19 Visentini PJ, Khan KM, Cook JL, et al. The VISA score: an index of the severity of jumper's knee (patellar tendinosis). F Sci Med Sport 1998;1:22-8.
20 Kirkley A, Griffin S, McLintock H, et al. The development and evaluation of a disease-specific quality of life measurement tool for shoulder instability. The Western Ontario Shoulder Instability Index (WOSI). Am f Sports Med 1998; 26:764-72.

21 Khan KM, Visentini PJ, Kiss ZS, et al. Correlation of US and MR imaging with clinical outcome after open patellar tenotomy: prospective and retrospective studies. Clinical fournal of Sport Medicine 1999;9:129-37.

22 Coleman BD, Khan KM, Kiss ZS, et al. Outcomes of open and arthroscopic patellar tenotomy for chronic patellar tendinopathy: a retrospective study. Am $\mathcal{F}$ Sports Med 2000; 28:183-90.

23 Cook JL, Khan KM, Kiss ZS, et al. Prospective imaging study of asymptomatic patellar tendinipathy in elite junio basketball players. F Ultrasound Med 2000;19:473-9.

24 Bellamy N. Pain assessment in osteoarthritis: experience with the WOMAC osteoarthritis index. Semin Arthritis Rheum 1989;184(suppl 2):14-17.

25 Huskisson EC. Measurement of pain. Lancet 1974;2:112731.

26 Melzack R. Concepts of pain measurement. In: Melzack R, ed. Pain measurement and assessment. New York: Raven Press, 1983:293.

27 Thordarson DB, Krieger LE. Operative vs. nonoperative treatment of intra-articular fractures of the calcaneus: a prospective randomized trial. Foot Ankle Int 1996;17:2-9.

28 Huskisson EC, Jones J, Scott PJ. Application of visual analogue scales to the measurement of functional capacity. Rheumatol Rehab 1976;15:185-7.

29 Flandry F, Hunt JP, Terry GC, et al. Analysis of subjective knee complaints using visual analog scales. Am f Sports Med 1991;19:112-18.

30 Scott PJ, Huskisson EC. Measurement of functional capacity with visual analogue scales. Rheumatol Rehab 1977;16: $257-9$.

31 Downie WW, Leathman PA, Rhind VM, et al. Studies with pain rating scales. Ann Rheum Dis 1978;37:378-81.

32 Price DD, McGrath PA, Rafii A, et al. The validation of visual analogue scales as ratio scale measures for chronic and experimental pain. Pain 1983;17:45-56.

33 Harrison E, Magee D, Quinney H. Development of a clinical tool and patient questionnaire for evaluation of patellofemoral pain syndrome patients.Clinical fournal of Sports Medicine 1996;6:163-70.

34 Lian O, Holen KJ, Engebrestson L, et al. Relationship between symptoms of jumper's knee and the ultrasound characteristics of the patellar tendon among high level male volleyball players. Scand F Med Sci Sports 1996;6:291-6.

35 Shalaby M, Almekinders LC. Patellar tendinitis: the significance of magnetic resonance imaging findings. Am F Sports Med 1999;27:345-9.

36 Cook JL, Kiss ZS, Khan KM. Patellar tendinitis: the significance of magnetic resonance imaging findings [letter]. Am 7 Sports Med 1999;27:831.

37 Percy EC, Conochie LB. The surgical treatment of ruptured tendo achillis. Am $\mathcal{F}$ Sports Med 1978;6:132-6.

38 Curwin S, Stanish WD. Tendinitis: its etiology and treatment. Lexington: Collamore Press, 1984.

39 Thermann H, Zwipp H, Tscherne H. Functional treatment concept of acute rupture of the Achilles tendon. 2 years results of a prospective randomized study. (In German.) Unfallchirurg 1995;98:21-32.

40 Boyden EM, Kitaoka HB, Cahalan TD, et al. Late versus early repair of Achilles tendon rupture. Clinical and biomechanical evaluation. Clin Orthop 1995;317:150-8.

41 Harms-Ringhdahl K, Carlsson AM, Ekhom J, et al. Pain assessment with different intensity scales in response to loading of joint structures. Pain 1986;27:401-11.

42 Jozsa L, Kvist M, Balint BJ, et al. The role of recreational sport activity in Achilles tendon rupture. A clinical, pathoSports Med 1989;17:338-43.

43 Hoher J, Munster A, Klein J, et al. Validation and application of a subjective knee questionnaire. Knee Surg Sports Traumatol Arthrosc 1995;3:26-33.

44 Brukher P, Khan K. Clinical Sports Medicine. Sydney: McGraw-Hill, 2001:547-8.

\section{Take home message}

There is a need for a quantitative index of pain and function in patients with Achilles tendinopathy. The VISA-A questionnaire is a valid, reliable, and easy to administer measure of the severity of Achilles tendinopathy and appears to be suitable for both clinical rating and quantitative research. 